\title{
What future for academic ophthalmology?
}

Has ophthalmology in the UK ever before faced such a range of challenges as those posed in the countdown to the new millennium? Technological developments in minimally invasive (day) surgery and the perceived benefits of community or 'near patient' care dictate that our major ophthalmic centres, whether in teaching hospitals or district general hospitals, will no longer be the main focus for our clinical activities. NHS research and development initiatives in health technology assessment, whereby new products and techniques will be introduced into clinical practice only after formal evaluation, will ensure evidence of clinical effectiveness in an increasingly knowledge based service, but will restrict individual specialists' traditional freedom to innovate. The implementation of the Calman Report will result in foreshortened and more intensive postgraduate training programmes (towards certification of completion of surgical training - CCST) but with the emergence of units and specialists with no junior staff or training role. This previously unimaginable development is already with us, witness the independent itinerant ophthalmologists who, exploiting the 'constructive tensions' (sic) of the internal NHS market and unfettered by training obligations, are competing with established ophthalmic institutions for their elective practice. They are also exposing the non-competitiveness of the private cataract surgery market, and UK ophthalmology (whether in the increasingly privatised NHS or in the independent sector) cannot expect to be spared in the global pursuit of medical cost containment. Against this background of profound change in the NHS and postgraduate training, academic departments of ophthalmology must also contend with the implications of the research selectivity exercise whereby departmental income, cascading through the university from the Higher Education Funding Council, will be inexorably linked with grant income and the quantity and quality of research output. Issues of academic survival will focus attention on the role of clinical academic trainees, whose distinguishing features from career registrars and senior registrars are more apparent than real (not least because of the imperative to complete their clinical training) and whose research contribution is often minimal.

What then are the implications of all this for academic ophthalmology in the UK? Firstly, we need to enhance and expand the research role of academically inclined clinical ophthalmologists after their completion of the CCST, possibly through lecturer appointments with honorary consultant status; clinical skills can thereby be maintained and developed but not at the expense of research output. Secondly, academic isolation must be overcome through even closer collaboration with the biological sciences (for biomedical research to prosper), through embracing academic optometry and other vision science resources, and through reaching out to the district hospitals and the community (to enhance the health services research capability). While the molecular and genetic sciences are improving our understanding of ophthalmic diseases and providing new methods of diagnosis and treatment for evaluation, academic ophthalmology will also have a major role to play in epidemiology and health needs assessment, in comparing the effectiveness of different patterns of service delivery, and in standard setting for (and coordination of) the increasingly fragmented service. Inherently tolerant of biological uncertainties, academic ophthalmology must grasp the opportunities proffered by a health care system in perpetual change.

Currently, recruitment into ophthalmology in the UK is of a very high quality, but how do we attract and keep the ablest within the academic fold? University vice chancellors are looking for (presumably non-pecuniary) incentives and rewards for those who are successful in the research market. So, with personal financial disincentives, differentials, and distractions in decline and with the threat of specialists' subjugation to managed care ordained by purchasers, won't the excitement of research and leadership in clinical development suffice?

DAVID MCLEOD

University Department of Ophthalmology, Manchester M13 9WH 